\title{
ANTECEDENTES DE LA POSTURA EMPRENDEDORA Y SU INFLUENCIA EN EL DESEMPEÑO EXPORTADOR DE LAS PYMES EN COLOMBIA*
}

\author{
Andrea Hurtado-Ayala** \\ Diana Marcela Escandón-Barbosa****
}

* doi: 10.11144/Javeriana.cao29-53.apei. Este artículo es producto de la investigación “Desarrollo Exportador en las Empresas Born Global en Colombia", financiado por la Pontificia Universidad Javeriana, Cali desde febrero de 2011 hasta diciembre de 2011. El artículo se recibió 26-04-2012 y se aprobó el 10-10-2016. Sugerencia de citación: Hurtado-Ayala, A. y Escandón-Barbosa, D. M. (2016). Antecedentes de la postura emprendedora y su influencia en el desempeño exportador de las pymes en Colombia. Cuadernos de Administración, 29 (53), 147-179. http://dx.doi.org/10.11144/Javeriana.cao29-53.apei.

* * Candidata al Doctorado en Administración de la Universidad del Valle, Cali, Colombia. Correo electrónico: anday490@yahoo.com

*** Doctora en Administración de la Universidad del Valle, Cali, Colombia, 2015. Docente de la Facultad de Ciencias Económicas y Administrativas de la Pontificia Universidad Javeriana, Cali, Colombia.

Correo electrónico: dmescandon@javerianacali.edu.co 


\section{Antecedentes de la postura emprendedora \\ y su influencia en el desempeño exportador de las pymes en Colombia}

\section{Resumen}

En este artículo se prueba que la postura emprendedora de las pymes influye positivamente sobre el desempeño exportador. Se utiliza la base de datos del Global Entrepreneurship Monitor (GEM) del 2011 para una muestra de 320 pymes exportadoras. Por medio de un modelo de ecuaciones estructurales se comprueba un efecto positivo de las características del emprendedor, características internas y externas y acceso a recursos gubernamentales sobre la postura emprendedora. Con estos resultados las pymes pueden reconocer sus debilidades y fortalezas sobre los recursos internos y su entorno, y poder tomar medidas para mejorar su desempeño exportador.

Palabras claves: internacionalización, pymes, emprendimiento, desempeño. Clasificación JEL: F13, F23, L19

Antecedents of the entrepreneurial stance and its influence on the export performance in Colombia SMEs

\author{
Antecedentes da postura \\ empreendedora e sua \\ influência no desempenho \\ exportador das PME na \\ Colômbia
}

\section{Abstract}

In this paper it is proven that the entrepreneurial stance on SMEs has a positive influence on export performance. The Global Entrepreneurship Monitor (GEM) database from 2011 is used for a sample of 320 exporting SMEs. Using structural equation modeling, a positive effect is proven of entrepreneurial characteristics, internal and external, and access to government resources on entrepreneurial stance. With these results, the SMEs can recognize their weaknesses and strengths on internal resources and their environment, and take steps to improve their export performance.

Keywords: Internationalization, SMEs, entrepreneurship, performance. JEL Classification: F13, F23, L19

\section{Resumo}

Neste artigo prova-se que a postura empreendedora das PME influi positivamente no desempenho exportador. Utiliza-se o banco de dados do "Global Entrepreneurship Monitor" (GEM) de 2011 para uma amostra de 320 PME exportadoras. Por meio de um modelo de equações estruturais, comprova-se um efeito positivo das características do empreendedor, características internas e externas e acesso a recursos governamentais sobre a postura empreendedora. Com esses resultados as PME podem reconhecer suas debilidades e fortalezas sobre os recursos internos e seu meio, e podem tomar medidas para melhorar seu desempenho exportador.

Palavras-chave: internacionalização, PME, empreendimento, desempenho.

Classificação JEL: F13, F23, L19 


\section{Introducción}

Las pequeñas y medianas empresas (pymes) dinamizan el desarrollo económico de los países, y en especial en los países emergentes, es por esto que la política económica en un país centra grandes esfuerzos en implementar e incentivar procesos de internacionalización que permitan un alto desempeño competitivo respecto a los mercados internacionales (Cancino y Bonilla, 2009; Belso, 2003), teniendo como foco central la promoción exportadora de las pymes por constituir un eje fundamental que promueve la generación de empleo y el crecimiento económico.

Existen diversos factores que parten de los enfoques teóricos sobre la internacionalización de empresas, y permiten entender como las empresas obtienen resultados superiores en el mercado internacional. Para ello, las teorías de internacionalización de la empresa se integran con la literatura sobre emprendimiento y rendimiento internacional, con el propósito de identificar los factores de éxito de las empresas en sus actividades exportadoras (Knudsen y Madsen, 2002).

A partir de esta integración se hace referencia a la iniciativa empresarial como proceso que conduce al éxito exportador de las pymes (Jennings y Lumpkin, 1989; Covin y Slevin, 1991; Zahra, 1993; Lumpkin y Dess, 1996), sustentando que el alto dinamismo y competitividad del mercado exportador con respecto al mercado nacional, crean la necesidad de examinar el vínculo entre emprendimiento y rendimiento, $\mathrm{y}$ la adopción de una postura emprendedora se convierte en un requisito para las empresas que buscan obtener un desempeño exportador satisfactorio (Covin y Slevin, 1989; Lumpkin y Dess, 1996). Por esta razón, esta investigación tiene dos objetivos: primero, identificar los antecedentes de la postura emprendedora de las pymes y examinar cómo estos factores afectan la adopción de dicha postura emprendedora, y segundo, determinar el impacto de una postura emprendedora sobre el desempeño exportador.

Este artículo da continuidad al estudio realizado por Escandón y Hurtado (2014), el cual analiza los factores determinantes del desarrollo exportador de las empresas Born Global en Colombia. Dicho estudio utilizó la base de datos GEM del año 2010 para analizar la influencia de variables como características internas, características del sector, características del entorno e innovación sobre el desarrollo exportador. En el presente artículo se tomó la base de datos GEM para 2011 para analizar las pymes exportadoras en general, se definió la postura emprendedora como variable que afecta el desempeño exportador y se analizaron los antecedentes de dicha postura. 
El desarrollo de este artículo se organiza de la siguiente forma: primero, se hace una revisión literaria sobre los factores determinantes de la postura emprendedora y el desempeño exportador. Segundo, se presenta el modelo conceptual para el estudio y se explican las relaciones entre los constructos del modelo propuesto. Posteriormente, se presenta la metodología de ecuaciones estructurales y los resultados obtenidos. Finalmente, se exponen las principales conclusiones del estudio

\section{Revisión literaria}

La internacionalización de empresas es una estrategia que conduce a la ampliación hacia mercados diferentes al local, mediante la constitución de diversas relaciones y procesos de adaptación a diferentes entornos (Alonso y Donoso, 1998; Coviello y McAuley, 1999; Rialp y Rialp, 2005). En términos generales, los estudios relacionados con las posibilidades con las que cuenta una empresa para internacionalizarse inician desde la década de 1970. Hedlund y Kverneland (1985) demuestran que existen dos formas altamente diferenciadas que permiten a las empresas internacionalizarse (internacionalización por procesos e internacionalización temprana), aunque la gran mayoría utiliza los modelos por procesos como la opción de internacionalización más segura y con mejores resultados.

En la década de 1970 algunos autores analizaron la internacionalización como un proceso gradual (Johanson y Wiedersheim-Paul, 1975; Lee y Brasch, 1978). Según Rialp y Rialp (2007) la internacionalización es un proceso dinámico gradual en el que las empresas (grandes y pequeñas) salen al extranjero y van acumulando experiencia y conocimiento de forma progresiva. Johanson y Vahlne $(1977,1990,2003)$ describen a las empresas con internacionalización gradual como aquellas centradas en los mercados nacionales durante un periodo prolongado, después del cual deciden entrar a mercados con características similares (Bell et al., 2003). Posteriormente, surge el emprendimiento internacional que explica la forma en que las pymes tienen un rápido éxito en el mercado internacional sin pasar por las diferentes etapas sugeridas en los modelos de internacionalización gradual (Peng, 2001). El emprendimiento internacional es definido como "una combinación de conducta innovadora, proactiva y arriesgada que cruza las fronteras nacionales e intenta crear valor en las organizaciones" (McDougall y 0viatt, 2000, p. 903). Con el emprendimiento internacional se explica la aparición de las nuevas empresas internacionales que hacen parte de la evolución del proceso de internacionalización presentado desde la década de 1970 y que actualmente resalta el desafío de algunas empresas frente a los procesos tradicionales de internacionalización (Rialp et al., 2005). El emprendimiento ha favorecido el desarrollo económico al contribuir a la identificación de nuevas opor- 
tunidades de negocio, creación de nuevas empresas, mejoramiento de sus procesos o impulso de la innovación y la competencia. Por esto, la literatura reciente ha centrado el interés en este tema y los investigadores no solo estudian las grandes compañías multinacionales sino que han incluido también las pequeñas empresas emprendedoras como unidad de análisis para estudiar el incremento del comercio internacional creado por la liberalización de los mercados y el desarrollo de las tecnologías de la información.

El crecimiento económico de los países se asocia con su estructura productiva, y en este caso, las pymes juegan un papel importante por su potencial para generar cambios en el mercado (Moori et al., 2005), y su contribución en el total de exportaciones de un país permite diversificar los tipos de productos exportados, favoreciendo los procesos de internacionalización, aunque también se evidencia que el desempeño exportador para este tipo de empresas se torna difícil ante la existencia de barreras competitivas que obstaculizan su permanencia en mercados externos. Por esta razón, se han realizado muchos estudios sobre el desempeño exportador de las pymes en diferentes países (McDougall y 0viatt, 2000; Cadogan et al., 2001, 2002, 2003 y 2006; Álvarez et al., 2005), y en Colombia se han desarrollado estudios sobre el perfil que deben tener las pymes para alcanzar un alto desempeño exportador (Moori et al., 2004, 2005).

En los estudios relacionados con las pymes exportadoras se establecen los diferentes mecanismos y procesos para la inserción internacional, encontrando algunos patrones de expansión para la internacionalización empresarial donde las pymes poseen algunos factores que se consideran determinantes en la decisión de implementar estrategias de internacionalización, entre los que se encuentran:

1. El direccionamiento hacia la innovación y la existencia de una estrategia de exportación (Moori et al., 2005).

2. El incremento de las opciones de financiación para implementar la estrategia exportadora (Moori et al., 2005).

3. Las capacidades organizacionales son una fuente de ventaja competitiva (Prahalad y Hamel, 1990; Galbreath, 2005; Knight y Cavusgil, 2004; Moori et al., 2005).

Las revisiones de la literatura sobre los determinantes del desempeño exportador se encuentran en Katsikeas et al. (1996), Zou y Stan (1998), Sousa et al. (2008), Zhang et al. (2013), Behyan (2015), Kim y Suh (2015). Estos estudios sobre internacionalización suponen que las variables que ejercen influencia sobre el desempeño exportador son: ventaja competitiva única, recursos destinados a la exportación, las características de gerentes y/o 
tomadores de decisión, la percepción de las oportunidades de exportación, y las características de las empresas (Cavusgil y Naor, 1987; Schlegelmilch y Crook, 1988; Sousa et al., 2008 y Houthoofd y Hendrickx, 2012; Escandón y Hurtado, 2014). Sin embargo, dada la importancia del espíritu empresarial en el desempeño de las exportaciones de las pymes, algunos estudios vinculan el emprendimiento con las exportaciones (Zahra et al., 1997; Caruana et al., 1998, Mostafa, 2005; Ismail et al., 2013; Fernández-Mesa y Alegre, 2015).

Shane y Venkataraman (2000) señalan que el "entrepreneurship" o emprendimiento es un proceso empresarial que identifica y evalúa oportunidades, y generalmente conduce a la creación de una nueva actividad económica o una nueva organización. Desde este punto de vista, los investigadores no solo estudian las grandes compañías multinacionales, sino que han incluido también las empresas emprendedoras como unidad de análisis, teniendo en cuenta el incremento del comercio internacional y la internacionalización de pymes, producida por la liberalización de los mercados y el desarrollo de las tecnologías de la información, llegando al surgimiento del concepto "emprendimiento internacional" definido como una combinación de conducta innovadora, proactiva y arriesgada que traspasa las fronteras nacionales e intenta crear valor en las organizaciones (McDougall y Oviatt, 2000). En el campo del emprendimiento internacional se especifica que la dinámica de los mercados internacionales requiere el estudio del vínculo entre emprendimiento y desempeño exportador, teniendo en cuenta la necesidad de una postura emprendedora para poder contar con procesos de exportación exitosos (Zahra et al., 1997; Covin y Slevin, 1989; Lumpkin y Dess, 1996).

Por lo tanto, partiendo del modelo planteado por Balabanis y Katsikeas (2003), el desempeño exportador se ve afectado por el grado de postura emprendedora adoptado por la empresa, y a su vez, la postura emprendedora se ve afectada por unos factores internos y externos, actuando como una variable moderadora entre estos factores y el desempeño exportador. Dentro de las variables que influyen en la postura emprendedora se consideran el tamaño de la empresa, la edad y su estructura organizativa como factores internos (Hansen, 1992; Covin y Slevin, 1988; Balabanis y Katsikeas, 2003); y la hostilidad, el dinamismo y la heterogeneidad son considerados como factores externos (Covin y Slevin, 1991; Zahra, 1991, 1993; Zahra et al., 1997; Balabanis y Katsikeas, 2003).

La internacionalización suele tener características diferentes según el tipo de empresa, por consiguiente, en este trabajo se analiza dicha estrategia en las pymes porque estas empresas desempeñan un importante papel en la internacionalización de los mercados y contribuyen en gran medida en la actividad exportadora de los países (Lu y Beamish, 
2001). Este artículo aborda los siguientes grupos de variables como factores antecedentes de la postura emprendedora: características del emprendedor, características internas y externas de la empresa, así como también recursos gubernamentales, consideradas como variables estratégicas relevantes.

\section{Modelo conceptual e hipótesis}

Este artículo se centra en estudiar los factores determinantes del desempeño exportador de las pymes colombianas: Características del emprendedor, Características Internas, Características Externas y Recursos Gubernamentales, presentando como variable moderadora la postura emprendedora de la empresa. El modelo conceptual propuesto para este estudio se muestra en la figura 1.

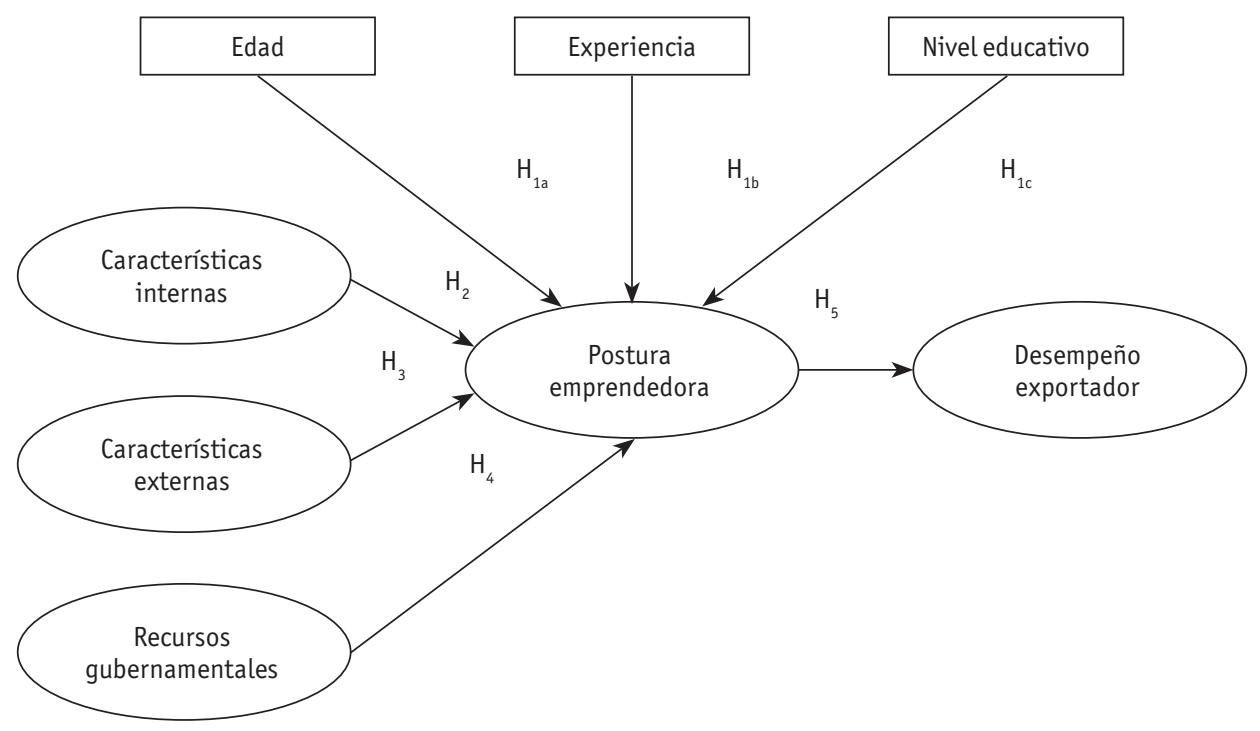

Figura 1. Modelo Conceptual

Fuente: elaboración propia.

\subsection{Características del emprendedor y postura emprendedora}

Cuando se hace referencia al emprendedor se piensa en el directivo que enfrenta una lucha contra la incertidumbre del entorno y decide ejercer acciones empresariales. Los siguientes estudios consideran que las características de los directivos de las empresas logran explicar cómo ellos superan las limitaciones que se presentan en los procesos de 
internacionalización: Aaby y Slater (1989), Coviello y Munro (1997), Madsen y Servais (1997), Westhead et al. (2001), Oviatt y McDougall (2005), Kuivalainen et al. (2007), Acedo y Jones (2007), Laanti et al. (2007), Karra et al. (2008), Kim y Hemmert (2016). Dichas características tales como la experiencia internacional, edad, género, idioma, educación, permiten que el empresario desarrolle estrategias globales que permiten reconocer las oportunidades del mercado (Jolly et al., 1992; Fillis, 2001; Leonidou et al., 1998, Gedik et al., 2015, Francioni et al., 2015; Laguir y Den Bester, 2016). Por lo tanto, las experiencias y habilidades del emprendedor explican el comportamiento empresarial y conducen a un desempeño exportador exitoso al brindar mayor conocimiento a la empresa (Storey, 1994; Escandón y Hurtado, 2012).

De acuerdo con Shrader et al. (2000) y Westhead et al. (2001), la experiencia previa del emprendedor consiste en un conjunto de habilidades que se adquieren de acuerdo con la edad y nivel educativo, y que influye positivamente sobre la actividad exportadora de la empresa donde se desempeñe. La edad del emprendedor refleja el tiempo en que ha adquirido su experiencia (Andersson et al., 2004) y el nivel educativo favorece el incremento de las posibilidades de crear empresa o realizar acciones estratégicas que conduzcan a mejores resultados empresariales (Gnywali y Fogel, 1994; Shane y Khurana, 2003; Shane, 2004; Audretsch et al., 2006; Ding y Stuart, 2006).

Las características y habilidades del emprendedor no son suficientes para conducir a un buen desempeño exportador, si la empresa no asume también una postura emprendedora (Lyon et al., 2000; De Clercq et al., 2013), es decir, un espíritu empresarial a nivel organizacional (Miller, 1983) que refleje cómo la empresa toma decisiones estratégicas bajo riesgo (Covin y Slevin, 1991; Zahra, 1993; Lumpkin y Dess, 1996) y tiene una inclinación a competir agresivamente con otros rivales (Balabanis y Katsikea, 2003). De acuerdo con el modelo planteado y a la evidencia empírica mencionada, la postura emprendedora afecta positivamente al desempeño exportador, pero esta postura emprendedora está influenciada por una serie de factores tanto internos como externos, de allí que la primera hipótesis planteada es:

Hipótesis 1a: Las características del emprendedor asociado a la edad, experiencia y nivel educativo influyen positivamente sobre la postura emprendedora de las pymes exportadoras colombianas.

Hipótesis 1b: Las caracteristicas del emprendedor asociado a la experiencia influyen positivamente sobre la postura emprendedora de las pymes exportadoras colombianas. 
Hipótesis 1c: Las características del emprendedor asociado al nivel educativo influyen positivamente sobre la postura emprendedora de las pymes exportadoras colombianas.

\subsection{Características internas de la empresa y postura emprendedora}

Knight y Cavusgil (1996) y Madsen y Servais (1997) consideran que el estudio del desempeño de las pymes exportadoras requiere del análisis de factores como el tamaño organizacional y los nichos de mercado, que constituyen características internas de una organización que afectan sus procesos de internacionalización (Westhead et al., 2001). Las características internas de la empresa son todos aquellos factores estructurales y decisiones de gestión influenciadas por las directivas para enfrentar los cambios en el mercado (Ferrier, 2001), especificando el grado en que los recursos organizacionales y de gestión son asignados en una empresa. En el caso de las pymes, se afirma que estas empresas por ser más pequeñas cuentan con mayor flexibilidad para adaptarse a los mercados exteriores, además, poseen un número reducido de empleados con respecto a las empresas grandes, facilitando procesos de comunicación efectivos y una coordinación de actividades mucho más eficiente (Covin y Slevin, 1989). Estas ventajas de la estructura interna de las pymes también favorecen las actividades de Investigación y Desarrollo implementadas generando un efecto positivo directo sobre los resultados empresariales (Cerrato, 2009; Morrison y Roth, 1992).

Estas características internas influyen en el desempeño exportador de las empresas (Aaby y Slater, 1989; Hollenstein, 2005), incluso autores como Zou y Stan (1998) señalan que son los factores que más afectan el rendimiento exportador dado que están bajo el control de las empresas. Sin embargo, teniendo en cuenta la existencia de la relación positiva entre postura emprendedora y desempeño exportador, la literatura plantea que las características internas de la empresa son factores determinantes de la adopción de una postura emprendedora que conduce al desempeño exportador (Ibrahim y Goodwin, 1986; Guth y Ginsberg, 1990; Covin y Slevin, 1991; Zahra, 1993; Lumpkin y Dess, 1996), de tal forma que las características internas son un antecedente de la postura emprendedora de las empresas (Guth y Ginsberg, 1990; Covin y Slevin, 1991; Zahra, 1993; Gnyawali y Fogel, 1994; Lumpkin y Dess, 1996). A partir de esta evidencia se plantean la siguiente hipótesis:

Hipótesis 2: Las características internas de la empresa como tamaño, generación de empleo e inversión en I+D influyen positivamente sobre la postura emprendedora de las pymes exportadoras colombianas. 


\subsection{Características externas y postura emprendedora}

A partir de la década de 1960 empezó a presentarse una mayor investigación sobre la interacción de la organización y el entorno (Cyerty March, 1963; Emery y Trist, 1965; Duncan, 1972; Starbuck, 1975). Entre los principales análisis de estos autores, se considera que las organizaciones deben tratar de enfrentar y adaptarse al entorno con el propósito de ser más eficaces y garantizar su supervivencia (Cyert y March, 1963; Thompson, 1967), señalando que las organizaciones pueden actuar en sus entornos con el fin de reducir la incertidumbre.

Desde el punto de vista del comportamiento exportador, otros autores se han centrado en clasificar los elementos que favorecen los procesos de internacionalización (Knighty Cavusgil, 1996; 2004; Madsen y Servais, 1997; Knight, 2000), encontrando que existe un grupo de factores externos conformado por el entorno internacional (Oviatty McDougall, 2005) y el sector al que pertenece la empresa (0viatty McDougall, 1995; Knighty Cavusgil, 1996; Madsen y Servais, 1997; Autio y Sapienza, 2000; Autio et al., 2000).

Las características externas son el resultado de las decisiones de gestión influenciadas por las fuerzas del mercado (Ferrier, 2001; Prietula y Watson, 2000). Por tanto, la capacidad de una empresa para adaptarse rápidamente a los mercados internacionales se asocia con su capacidad de aprender de las oportunidades del mercado y de los cambios del entorno (0zsomer y Gençtürk, 2003).

Al hacer referencia a las características externas que afectan el desempeño exportador, se encuentran: el sector económico al que pertenece la empresa (Bell, 1995; Oviatt y McDougall, 1995; Knight y Cavusgil, 1996; Autio et al., 2000, Blomqvist et al., 2008; Escandón y Hurtado, 2013), el nivel tecnológico del sector que puede conducir a procesos de internacionalización más rápidos y eficientes (Knight y Cavusgil, 1996; Bleaney y Wakelin, 2002; Autio et al., 2000; Oviatt y McDougall, 2005), y la intensidad competitiva o el grado de competencia que enfrenta una empresa en el mercado (Jap, 1999; Jaworski y Kohli, 1993; Shrader et al., 2000; Westhead et al., 2002; Escandón y Hurtado, 2013).

Los factores externos también son considerados como aspectos relevantes para el desarrollo de estrategias y la adopción de una postura emprendedora en las empresas exportadoras (Jain, 1989; Cavusgil y Zou, 1994; Balabanis y Katsikeas, 2003), al sugerirse que se podrían obtener mayores beneficios mediante la implementación de estrategias que respondan a las necesidades del mercado (Jain, 1989). Adicionalmente, el empren- 
dimiento se define como un fenómeno que se origina a partir del entorno (Morris y Paul, 1987), como consecuencia del dinamismo del mercado, la intensidad competitiva y la heterogeneidad del mercado (Covin y Slevin, 1991; Zahra, 1993; Zahra et al., 1997), por esta razón, las organizaciones enfrentan la incertidumbre del entorno adoptando una postura emprendedora (Miller et al., 1988; Dimitratos y Plakoyiannaki, 2003; Balabanis y Katsikeas, 2003), y en tanto el entorno de las empresas exportadoras es más competitivo y riesgoso, las empresas tienden a alinear dichas características externas con una postura emprendedora que conduzca a un alto desempeño exportador (Zahra et al., 1997; Albaum et al., 2001; Balabanis y Katsikeas, 2003). Partiendo de esta evidencia se plantea la tercera hipótesis.

Hipótesis 3: Las características externas de la empresa como el sector económico, la intensidad competitiva y nivel tecnológico del sector influyen positivamente sobre la postura emprendedora de las pymes exportadoras colombianas.

\subsection{Acceso a recursos gubernamentales y postura emprendedora}

La actividad exportadora es un proceso dinámico asumido por las empresas y que en la mayoría de los casos requiere de apoyo externo para lograr un éxito exportador (Cannon y Willis, 1983 y Rosson y Seringhaus, 1991). Según el tamaño las empresas pueden necesitar diferentes tipos de ayuda para afrontar su actividad exportadora, y desde este punto de vista, el apoyo más común es de carácter financiero (Pauwels y Matthyssens, 1999). En Colombia, las pymes suelen recurrir al financiamiento de entidades privadas sector público, y apoyo de acreedores y clientes (Salazar, Cabrera, y Becerra, 2011). Bajo estas circunstancias, las políticas económicas nacionales requieren que las instituciones gubernamentales se adapten al contexto y establezcan un mayor grado de interacción con los actores claves para el desarrollo económico (Yáñez et al., 2008). Por esto la promoción a las exportaciones por parte del sector público está dirigida especialmente a las pymes, con el fin de apoyar su entrada y permanencia en los mercados extranjeros (Geldres et al., 2011), mediante programas de apoyo a disposición de las pymes para incrementar el acceso de estas empresas al mercado internacional e incrementar los resultados de las exportaciones.

En general, los acuerdos de financiación patrocinados por el gobierno y financiados con fondos públicos estimulados por los programas gubernamentales de investigación y desarrollo, permiten a las empresas ampliar su mercado (Andersson et al., 2004; Rialp-Criado et al., 2002; Matt y Wolff, 2004) e impactar positivamente sobre el desempeño exportador 
dado que generan ventajas competitivas ante la posibilidad de adaptarse más rápidamente a las necesidades del mercado internacional (Kotabe y Czinkota, 1992).

La relación entre la promoción exportadora mediante recursos gubernamentales y el desempeño exportador ha sido estudiada por autores que han comprobado la existencia de una relación positiva entre estas dos variables (Moini, 1998; Gençtürk y Kotabe, 2001; Lages y Montgomery, 2005; Geldres et al., 2009). Dada la evidencia encontrada acerca de la relación entre postura emprendedora y desempeño exportador (Covin y Slevin, 1991; Balabanis y Katsikea, 2003), en este artículo se considera que siendo la postura emprendedora una habilidad empresarial de asumir riesgos con respecto a decisiones de inversión y acciones estratégicas dentro de un contexto de incertidumbre, la búsqueda de mayores oportunidades y de mayor competitividad a través del apoyo gubernamental favorecen las decisiones propiciadas por la misma postura emprendedora (Covin y Slevin, 1989; Miller y Friesen, 1982). Así, se plantea la cuarta hipótesis.

Hipótesis 4: El acceso a los recursos gubernamentales influye positivamente sobre la postura emprendedora de las pymes exportadoras.

\subsection{Postura emprendedora y desempeño exportador}

El proceso de internacionalización hace parte de las estrategias empresariales de una organización (Andersson, 2000), razón por la cual se encuentran investigaciones que asocian el espíritu emprendedor con los negocios internacionales (McDougall y 0viatt, 2000), sustentando que la globalización de los mercados es una oportunidad para estudiar el impacto que tiene la orientación emprendedora sobre el desempeño exportador de las empresas (Zahra y Garvis, 2000), sin embargo no hay muchos estudios sobre el desempeño exportador desde el punto de vista del emprendimiento (Guth y Ginsberg, 1990).

Existe evidencia sobre el análisis de la relación entre postura emprendedora y desempeño exportador (Ibrahim y Goodwin, 1986; Covin, 1991; Covin y Slevin, 1991; Zahra, 1993; Herron y Robinson, 1993; Lumpkin y Dess, 1996; Li et al., 2008; Frishammar y Hörte; 2007; Balabanis y Katsikea, 2003; Grinstein, 2008, Etchebarne et al., 2010), y la mayor parte de la investigación en el área indica una relación positiva entre ambas variables. La postura emprendedora u orientación empresarial se define como la gestión de tomar decisiones de inversión riesgosas y emprender acciones estratégicas en un contexto de incertidumbre, ampliando la innovación de productos, el liderazgo tecnológico y la propensión de la empresa a competir proactivamente (Covin y Slevin, 1991). La adopción de 
una postura emprendedora posibilita el aumento del rendimiento de las exportaciones, porque incrementa la eficacia de la gestión empresarial (Covin y Slevin, 1991; Ibrahim y Goodwin, 1986; Guth y Ginsberg, 1990; Herron y Robinson, 1993; Lumpkin y Dess, 1996; Balabanis y Katsikea, 2003; Grinstein, 2008, Etchebarne et al., 2010) y propicia el ingreso temprano de empresas al mercado internacional (Cooper, 1979; Cahill, 1996). Adicionalmente, permite crear una ventaja competitiva porque las empresas con una postura emprendedora están en la capacidad de responder a los cambios del entorno con oportunidades obtenidas a partir de la proactividad y la habilidad para crear nuevos productos y establecerse en nuevos mercados (Blesa y Ripollés, 2004). Las empresas con estrategias basadas en una postura emprendedora pueden alcanzar un mejor desempeño empresarial que aquellas empresas que no asumen tal postura, y adoptan otro tipo de estrategia empresarial para canalizar los factores internos y externos que afectan el desempeño exportador (Frishammar y Hörte, 2007; Grinstein, 2008). Por lo anterior, se plantea la quinta hipótesis.

Hipótesis 5: La adopción de una postura emprendedora influye positivamente sobre el desempeño exportador de las pymes exportadoras.

\section{Metodología}

Teniendo en cuenta la actividad exportadora en Colombia en el 2011, el intercambio comercial de Colombia con otros países tuvo un incremento de $39,4 \%$ con respecto al año 2010, de dicho incremento el 52,3\% se le atribuye a las exportaciones (Dian, 2012). Las exportaciones se incrementaron en $43 \%$ con respecto al año 2010 , en especial por el incremento en las exportaciones de petróleo y sus derivados, así como la participación de la venta de productos manufacturados, productos de la floricultura, azúcares y confitería y frutos comestibles. En el 2011, el valor exportado fue el valor histórico más alto desde 2007 (US\$56.954 millones), dado que durante el periodo 2010 a 2013 con el incremento de los acuerdos comerciales se produjo la recuperación de las exportaciones tras la desaceleración económica del 2008 (MinCIT, 2014). En este mismo periodo las pymes exportadoras vendieron al exterior 1.193 veces más que las empresas grandes, y dichas pymes alcanzaron un incremento en el número de empleados de $6,1 \%$. No obstante, su participación en la actividad exportadora sigue siendo pequeña, teniendo en cuenta que estas empresas realizan el $5 \%$ de las ventas colombianas al exterior (Lacouture, 2015).

La población que será estudiada corresponde a pymes exportadoras colombianas. Para probar las hipótesis planteadas se realizó un estudio cuantitativo con la base de datos 
Global Entrepreneurship Monitor (GEM) Colombia 2011. Esta base de datos está construida a partir de encuestas realizadas por el Centro Nacional de Consultoría (CNC), en cinco ciudades capitales (Bogotá, Medellín, Cali, Barranquilla y Bucaramanga), tres ciudades capitales de departamento (Santa Marta, Cúcuta y Pasto) y 23 municipios adicionales, seleccionados aleatoriamente y que cumplen con la condición de poseer una población inferior a 10.000 habitantes. Filtrando la base de datos de acuerdo con las condiciones de tamaño empresarial descritas en la revisión literaria de este artículo se encuentra una muestra de 320 pymes exportadoras.

La tabla 1 muestra la composición general de la muestra seleccionada para este estudio. Se resalta que existe un equilibrio de las frecuencias por tamaño empresarial y que tiende a ser acorde con el tejido empresarial colombiano vinculado a los mercados internacionales. Adicionalmente, se resalta que el $68,75 \%$ de las empresas son exportadoras tradicionales o que han llevado un proceso gradual de incursión en los mercados internacionales y un $31,25 \%$ son Born Global o empresas que se aventuran en sus tres primeros años a exportar. En términos de la antigüedad existe un predominio de empresas de empresas con menos de cinco años en el mercado (30\%) y solo un $18 \%$ con una antigüedad de más de 20 años.

\section{Tabla 1}

Estructura de la muestra

\begin{tabular}{llc}
\hline \multicolumn{1}{c}{ Características } & & Frecuencia (\%) \\
\hline \multirow{3}{*}{ Tamaño de la empresa } & Mipyme & 12,50 \\
& Pyme & 31,25 \\
& Mediana & 28,12 \\
Tipo de empresa exportadora & Grande & 28,12 \\
& Tradicional & 68,75 \\
& Born Global & 31,25 \\
Antigüedad de la empresa & Menor 5 años & 30,00 \\
& Entre 5 y 9 años & 23,40 \\
& Entre 10 y 19 años & 28,10 \\
& Más de 20 años & 18,50 \\
\hline
\end{tabular}

Fuente: elaboración propia.

Con el fin de proporcionar una visión general de las interrelaciones entre los seis constructos, en la tabla 2 se presentan las medidas, desviaciones estándary las correlaciones de cada una de las variables incluida en el estudio. En el caso de la matriz de correlaciones se puede apreciar que el valor medio de los diferentes constructos es de 4,38 y una 
desviación estándar de 1,20 permitiendo concluir la variablidad de respuestas dentro del cuestionario. Por otra parte, con respecto a los niveles de correlación se encontrar valores que permiten concluir que todos los constructos pueden ser usados en un modelo de ecuaciones estructurales pues el nivel de asociación son los adecuados y no generan problemas de heteroscedasticidad.

\section{Tabla 2}

Medias, desviaciones y correlaciones

\begin{tabular}{|c|c|c|c|c|c|c|c|c|}
\hline \multirow{2}{*}{ Constructos } & \multirow{2}{*}{ Media } & \multicolumn{7}{|c|}{ S. D. } \\
\hline & & & 1 & 2 & 3 & 4 & 5 & 6 \\
\hline 1. Postura emprendedora & 4,39 & 1,32 & 0,51 & & & & & \\
\hline 2. Desempeño exportador & 3,45 & 1,42 & 0,61 & 0,66 & & & & \\
\hline 3. Características del emprendedor & 5,09 & 1,50 & 0,69 & 0,58 & 0,59 & & & \\
\hline 4. Características internas & 4,28 & 1,24 & 0,35 & 0,29 & 0,33 & 0,51 & & \\
\hline 5. Características externas & 4,39 & 1,31 & 0,24 & 0,20 & 0,23 & 0,22 & 0,61 & \\
\hline 6. Recursos gubernamentales & 4,65 & 1,23 & 0,68 & 0,57 & 0,41 & 0,19 & 0,23 & 0,67 \\
\hline
\end{tabular}

Fuente: elaboración propia.

\subsection{Las variables del modelo}

Las características internas, características externas, características del emprendedor y los recursos gubernamentales permiten aumentar la Postura Emprendedora de las pymes exportadoras y su posible éxito en el mercado internacional. Para medir estos constructos se toman varios indicadores y escalas utilizadas en la encuesta GEM, equivalentes a algunas escalas que empíricamente han sido probadas en diversas investigaciones.

Postura emprendedora: una de las escalas más reconocidas es la desarrollada por Covin y Slevin $(1988,1989)$ conformada por una escala likert de nueve ítems que consta de los siguientes elementos que describen la iniciativa empresarial: innovación, proactividad y asunción de riesgos. En esta investigación se utilizan las siguientes variables equivalentes medidas en la base de datos GEM: se toma la variable innovación medida como el número de empresas que ofrecen productos o servicios diferentes. La proactividad se mide mediante una variable proxy denominada nueva actividad empresarial, y por último, la actitud frente al riesgo se mide a través del temor al fracaso empresarial dado como el porcentaje de la población que percibe que el miedo al fracaso podría disuadirlo a la hora de poner en marcha una empresa. 
Desempeño exportador: dentro de la gran cantidad de estudios relacionados con el desempeño exportador, se pueden identificar tres medidas de esta variable y que se relaciona con la forma de conceptualización del constructo como niveles de ventas internacionales (Cavusgil, 1984; Czinkota y Johnson, 1983; Madsen, 1998), crecimiento de las ventas internacionales (Johnson y Arunthanes, 1995) y volúmenes de exportación (Axinn, 1988). Las variables que se incluyen en esta medición son percepción de crecimiento, actividad exportadora satisfactoria, expectativas del nivel de ventas. Estas variables son medidas en una escala de 1 a 7 donde 1 es muy en desacuerdo y 7 muy de acuerdo, preguntando a los encuestados como evidencia el desempeño de su empresa durante los próximos meses con respecto a las posibilidades de: lograr un rápido crecimiento, rendimiento de las exportaciones e incremento en nivel de ventas.

Características del emprendedor: se refiere a las características personales y demográficas del empresario. Varios estudios encuentran que las características demográficas como la edad y el género son las variables que logran obtener un gran impacto en la intensión emprendedora y su posible desempeño (Reynolds et al., 2000; Sinha, 1996; Mazzarol et al., 1999). Por otra parte, las características personales como nivel educativo y experiencia en industrias similares son reconocidas como facilitadoras para el desarrollo de habilidades en los directivos (Cragg y King, 1988).

Características internas: las características internas desarrolladas en esta investigación incluyen variables como tamaño de las ventas, generación de empleo e inversión en investigación y desarrollo (I+D). El tamaño de las ventas se utiliza como proxy del tamaño empresarial y se asume en muchas ocasiones como prerrequisito para obtener altos niveles de exportación y de desempeño exportador (Reid, 1983). En términos de la generación de empleo se utilizan la evolución del número de puestos generados o su nivel de crecimiento y se mide dentro del GEM preguntando a los empresarios sobre si ellos creen que su empresa generará algún empleo ahora o en los próximos cinco años, y si su expectativa de crecimiento de la empresa se estudia mediante la identificación de cuántos empresarios esperan generar más de 19 puestos de trabajo.

Características externas: este constructo se incluye para analizar las condiciones del entorno de una empresa y se mide por niveles de intensidad competitiva del sector, niveles tecnológicos del sector y sector de actividad. La intensidad competitiva del sector esta medida por la percepción sobre los niveles de competencia que se tienen dentro del sector de actividad en el que se encuentra la empresa (Jap, 1999; Jaworski y Kohli, 1993; Shader et al., 2000; Westhead et al., 2002). El nivel tecnológico se mide por el nivel de tecnolo- 
gía utilizada y permite conocer qué tan dinámico es el sector en el que se encuentra la empresa (Knight y Cavusgil, 1996; Bleaney y Wakelin, 2002; Autio et al., 2000; 0viatt y McDougall, 2005). En esta investigación se mide como el tiempo que ha estado disponible la tecnología utilizada para la producción del bien o servicio que ofertan tanto los nuevos empresarios como los empresarios establecidos.

Por otra parte, el sector de actividad se establece de acuerdo con la Clasificación Industrial Internacional Uniforme (CIIU) en tres categorías: el sector extractivo (agricultura, forestal, pesca y minería), el sector de trasformación (la construcción, la manufactura, el transporte, las distribuciones y ventas al por mayor), y el sector de servicios industriales (seguros, bienes raíces y demás servicios a las empresas).

Las medidas utilizadas para las variables características del emprendedor, características internas y externas corresponden a las utilizadas en el estudio de Escandón y Hurtado (2014).

Recursos gubernamentales: los recursos gubernamentales que apoyan las exportaciones se miden mediante los acuerdos de financiación patrocinados por el gobierno, fondos públicos que estimulan la investigación y desarrollo, y monto de recursos públicos invertidos en apoyo exportador (Matt y Wolff, 2004). En esta investigación, el constructo se compone de los siguientes indicadores: financiación (montos monetarios para el desarrollo de actividades), redes con entidades gubernamentales (número de entidades con las que tiene acuerdos) y apoyo-asesoría gubernamental (frecuencia con la que la empresa ha solicitado asesoría empresarial a entidades gubernamentales).

\subsection{El modelo: ecuaciones estructurales}

Las ciencias sociales tienen dentro de su campo de estudio la necesidad de medir tanto conceptos no físicos como abstractos denominados constructos, y que son resultados indirectos de un grupo de indicadores asociados a su definición. En este sentido, los modelos de ecuaciones estructurales se convierten en un instrumento adecuado para el establecimiento de relaciones causales en diferentes vías entre dichos constructos. Para este fin se diseña el modelo teórico que expresa de forma abstracta la realidad inmersa en las variables latentes, definiendo las relaciones entre ellas. La hipótesis dentro de este modelo se representa en la estructura de varianzas y covarianzas de las variables del estudio. 


\section{Pruebas de FIABILIDAD DEL MODELO DE ECUACIONES ESTRUCTURALES}

Para analizar las propiedades psicométricas de las escalas utilizadas en este estudio se realizó un análisis factorial confirmatorio para evaluar la validez convergente y discriminante de los constructos. El análisis factorial indica que los datos presentan un ajuste razonable y todas las medidas muestran una fiabilidad adecuada medida a través de los índices de fiabilidad compuesta (SCR) que son mayores a 0,6 y la varianza media extraída (AVE) que es superior a 0,5 .

Además todas las cargas corresponden a sus factores hipotéticos y las estimaciones son muy significativas dado que el $t$-valor presenta valores altos sugiriendo validez convergente (Bagozzi y Yi, 1988). También se confirma la validez discriminante porque en cada escala se obtiene un promedio de la varianza extraída por el constructo subyacente mayor que la varianza compartida con otro constructo latente. Ver la tabla 3.

\section{Tabla 3}

Resumen de la medición de los constructos

\begin{tabular}{|c|c|c|c|}
\hline Descripción de ítems & $\begin{array}{c}\text { Carga } \\
\text { estandarizada }\end{array}$ & t-valor & $\begin{array}{c}\text { Fiabilidad } \\
\mathrm{SCR}^{\mathrm{a}} \\
\mathrm{AVE}^{\mathrm{b}}\end{array}$ \\
\hline \multicolumn{4}{|l|}{ Postura emprendedora } \\
\hline Innovación & 0,64 & 11,95 & $\mathrm{SCR}=0,80$ \\
\hline Nueva actividad empresarial & 0,68 & 12,93 & AVE $=0,51$ \\
\hline Temor al fracaso empresarial & 0,75 & 14,57 & \\
\hline \multicolumn{4}{|l|}{ Desempeño exportador } \\
\hline Percepción de crecimiento & 0,74 & 14,93 & $\mathrm{SCR}=0,89$ \\
\hline Actividad exportadora satisfactoria & 0,73 & 14,63 & $\mathrm{AVE}=0,66$ \\
\hline Expectativa del nivel de ventas & 0,90 & 20,15 & \\
\hline \multicolumn{4}{|l|}{ Características internas } \\
\hline Tamaño empresarial & 0,72 & 14,24 & $\mathrm{SCR}=0,88$ \\
\hline Generación de empleo & 0,76 & 15,40 & $\mathrm{AVE}=0,59$ \\
\hline Inversión en investigación y desarrollo & 0,78 & 15,95 & \\
\hline \multicolumn{4}{|l|}{ Características externas } \\
\hline Intensidad competitiva del sector & 0,60 & 10,35 & $\mathrm{SCR}=0,76$ \\
\hline Niveles tecnológicos del sector & 0,85 & 14,67 & $\mathrm{AVE}=0,51$ \\
\hline Sector de actividad & 0,68 & 11,88 & \\
\hline \multicolumn{4}{|l|}{ Recursos gubernamentales } \\
\hline Financiación & 0,76 & 14,55 & $\mathrm{SCR}=0,83$ \\
\hline Redes con entidades gubernamentales & 0,74 & 14,06 & $\mathrm{AVE}=0,61$ \\
\hline Apoyo asesoría gubernamental & 0,85 & 16,84 & \\
\hline
\end{tabular}




\begin{tabular}{lccc}
\hline \multicolumn{1}{c}{ Descripción de ítems } & $\begin{array}{c}\text { Carga } \\
\text { estandarizada }\end{array}$ & t-valor & $\begin{array}{c}\text { Fiabilidad } \\
\text { SCR }^{\text {a }} \\
\text { AVE }^{\text {b }}\end{array}$ \\
\hline Características del emprendedor & & & \\
Edad & 0,66 & 14,55 & SCR $=0,80$ \\
Experiencia & 0,72 & 14,06 & AVE $=0,59$ \\
Nivel educativo & 0,80 & 16,84 & \\
\hline
\end{tabular}

Notas: estadísticos de ajuste para el modelo de 18 indicadores y seis constructos: $\chi^{2}=799,82 ; \mathrm{GFI}=$ 0,$88 ;$ RMSEA=0,041; SRMR=0,047; CFI= 0,95; TLI (NNFI)=0,95. ${ }^{\text {a }}$ Escala de fiabilidad compuesta $\left(\rho c=\left(\sum \lambda i\right) 2 \operatorname{var}(\xi) /\left[\left(\sum \lambda i\right) 2 \operatorname{var}(\xi)+\sum \theta i i\right] ;\left(\right.\right.$ Bagozzi y Yi, 1998)). ${ }^{\text {b }}$ Promedio de la varianza extraída $\left(\rho c=\left(\sum \lambda i 2 \operatorname{var}(\xi)\right) /\left[\sum \lambda i 2 \operatorname{var}(\xi)+\sum \theta i i\right] ;(\right.$ Fornell y Larcker, 1981).

Fuente: elaboración propia.

Por último, la evaluación de la validez discriminante de constructos se comprueba si los valores de la matriz de correlaciones estandarizadas entre variables latentes no son superiores a 0,9 (Del Barrio y Luque, 2000) y logra ser evidente en esta investigación. Por tanto, se concluye que el modelo permite medir constructos diferenciados.

\section{Resultados}

El contraste de hipótesis son estimadas utilizando un modelo estructural del conjunto de hipótesis planteadas en este trabajo. Para dicha estimación se tomó como input la matriz de correlaciones y los resultados ponen de manifiesto que el modelo presenta un ajuste aceptable: $\chi^{2}(83)=226,58(p=0,00)$; CFI $=0,93$; NFI=0,91; SRMR= 0,079; RMSEA=0,08; $\mathrm{GFI}=0,91$. Los resultados obtenidos avalan todas las relaciones planteadas en el apartado teórico. La hipótesis 1a, 1b y 1c se confirma, las características del emprendedor (edad, formación profesional, experiencia) influyen positivamente sobre la postura emprendedora de las pymes exportadoras colombianas. La hipótesis 2 también se confirma porque las características internas de la empresa tales como el tamaño, la generación de empleo y la inversión en I+D están relacionados positivamente con la Postura Emprendedora de la empresa $\left(\gamma_{31}=0,90 p<0,01\right)$. En cuanto a las características externas de las pymes (Hipótesis 3$)$ se evidencia una relación positiva con la postura emprendedora asumida $\left(\gamma_{51}=0,76\right.$ $p<0,10)$. La hipótesis 4 se confirma, el acceso a recursos gubernamentales ejerce una influencia significativa sobre la postura emprendedora de las pymes. $\left(\gamma_{61}=0,63 p<0,10\right)$. En cuanto la hipótesis 5 , se confirma que la postura emprendedora afecta positivamente al desempeño exportador. $\left.\beta_{21}=0,49 p<0,01\right)$. 


\section{Tabla 4}

Resultados del modelo estructural

\begin{tabular}{|c|c|c|c|c|}
\hline \multirow{2}{*}{\multicolumn{2}{|c|}{$\begin{array}{l}\text { Hipótesis del modelo } \\
\text { Parámetro }\end{array}$}} & \multicolumn{3}{|c|}{$\begin{array}{c}\text { Estimación de los parámetros } \\
\text { estandarizados }\end{array}$} \\
\hline & & Estimado & T-Valor & \\
\hline Hipótesis 1 & $\begin{array}{l}\text { Las características del emprendedor influyen positivamente sobre } \\
\text { la postura emprendedora de las pymes exportadoras colombianas. }\end{array}$ & Y11 & 0,85 & 6,43 \\
\hline Hipótesis 2 & $\begin{array}{l}\text { Las características internas influyen positivamente sobre la } \\
\text { postura emprendedora de las pymes exportadoras colombianas. }\end{array}$ & $\Upsilon 31$ & 0,90 & 13,57 \\
\hline Hipótesis 3 & $\begin{array}{l}\text { Las características externas influyen positivamente sobre la } \\
\text { postura emprendedora de las pymes exportadoras colombianas }\end{array}$ & $\Upsilon 51$ & 0,73 & 8,92 \\
\hline Hipótesis 4 & $\begin{array}{l}\text { El acceso a recursos gubernamentales influyen positivamente } \\
\text { influyen positivamente sobre la postura emprendedora de las } \\
\text { pymes exportadoras colombianas }\end{array}$ & Y61 & 0,63 & 15,98 \\
\hline Hipótesis 5 & $\begin{array}{l}\text { La postura emprendedora influye positivamente sobre el } \\
\text { desempeño exportador de las pymes exportadoras colombianas }\end{array}$ & $\beta 21$ & 0,49 & 6,60 \\
\hline
\end{tabular}

Fuente: elaboración propia.

\section{Discusión de resultados}

En este estudio se encuentra que las pymes colombianas desarrollan diferentes aspectos característicos que afectan su desempeño exportador. Las características del emprendedor son centrales para desarrollar los procesos de internacionalización (Karra et al., 2008; Kim y Hemmert, 2016), esto se evidencia en las pymes colombianas en donde aspectos como la edad del empresario, su experiencia y nivel educativo son aspectos que incrementan sus habilidades directivas para la toma de decisiones favoreciendo su postura emprendedora, al aumentar las oportunidades para crear empresa y desarrollar actividades que conduzcan a un mayor desempeño (Ding y Stuart, 2006). Por ejemplo, en cuanto a la edad y experiencia del empresario pueden afectar el nivel de riesgo asumido en la toma de decisiones (Ding y Stuar, 2006), mientras que el nivel educativo unido a la experiencia favorecen la acumulación de conocimientos para tomar acciones estratégicas adecuadas y desarrollar las actividades exportadoras con mayor eficiencia. A nivel organizacional, las pymes exportadoras colombianas desarrollan factores internos y enfrentan factores externos que afectan su postura emprendedora; tal es el caso de aspectos como el tamaño de la empresa, la inversión en I+D y la generación de empleo que son aspectos controlados por la empresa, asociados con el nivel de recursos que son asignados para su funcionamiento. De igual forma, el entorno organizacional ejerce influencia sobre las decisiones empresariales conforme a aspectos como el sector económico, la intensidad 
competitiva del sector y su nivel tecnológico, de los cuales también depende la supervivencia de las pymes exportadoras colombianas.

En este sentido, las características internas y externas presentadas en este trabajo constituyen factores que determinan la postura emprendedora de las pymes exportadoras colombianas y que afectan también su desempeño exportador, dado que afectan las estrategias asumidas por la empresa para enfrentar el mercado (Balabanis y Katsikeas, 2003), e influyen en la eficiencia de las actividades internacionales que desarrollan estas empresas (Oviatt y McDougall, 2005).

Con respecto al acceso a los recursos gubernamentales, los resultados coinciden con los planteamientos de Andersson et al. (2004) y Matt y Wolff (2004) acerca del impacto positivo que tiene la financiación propiciada por el gobierno sobre el desempeño exportador. El acceso a los recursos gubernamentales por parte de las pymes colombianas les ha permitido ampliar su participación en el mercado extranjero, dado que dichos recursos constituyen una importante fuente de inversión que les permite tomar decisiones estratégicas en favor de la actividad exportadora, de tal forma que se favorece su postura emprendedora al poder asumir riesgos en mercados internacionales gracias al apoyo del gobierno en materia de recursos (Covin y Slevin, 1989).

Finalmente, los resultados de este estudio demuestran la influencia positiva de la postura emprendedora asumida por las pymes colombianas, sobre su desempeño exportador. Las características del empresario, las características internas y externas de las empresas, y el acceso a los recursos gubernamentales son aspectos que favorecen una postura emprendedora definida como la habilidad empresarial de tomar decisiones en un entorno de incertidumbre con el propósito de acceder a mayores oportunidades en el mercado y lograr mayor competitividad. Por tanto, dado que las pymes colombianas desarrollan dicha postura emprendedora, ésta a su vez mejora la gestión empresarial, aumenta las ventajas competitivas de la empresa otorgándole fortalezas para poder enfrentarse a nuevos mercados, alcanzando un incremento de su desempeño exportador.

\section{Conclusiones}

En este artículo se demuestra que tener altos niveles de postura emprendedora asociada a la presencia de características del empresario (características personales y demográficas), características internas (tamaño empresarial, generación de empleo e I+D), características externas (intensidad competitiva del sector, niveles tecnológicos del sector, sector de 
actividad) y recursos gubernamentales (financiación, redes entidades gubernamentales, apoyo-asesoría gubernamental), permite mejorar los niveles de desempeño exportador. Así, la postura emprendedora se convierte en un elemento clave para la ampliación de las exportaciones y un indicador sobresaliente del desempeño exportador.

Los resultados arrojan que existe una relación positiva entre las características del emprendedor como las de tipo personal y demográficas. Por tanto, en la literatura se asume que el empresario puede tener un determinado perfil que puede facilitarle su posibilidad de aumentar su postura emprendedora y consiguiente éxito en los mercados internacionales (Gomez-Mejia, 1988; Evangelista, 1994). Dentro de este constructo de características del emprendedor sobresalen las de tipo personal como las más influyentes en el desarrollo de las habilidades directivas y en su forma de enfrentarse al mercado internacional, especialmente el nivel educativo y experiencia en industrias similares.

Por otra parte, las características internas asociadas al tamaño empresarial, generación de empleo e inversión en investigación y desarrollo (I+D) se constituye en un constructo de alta importancia para el logro de una postura emprendedora de largo plazo y exitosa. Específicamente, el tamaño empresarial se asocia como un requerimiento para el logro de altos desempeños exportadores. Sin embargo, es importante resaltar que no necesariamente las empresas de gran tamaño obtienen mejores niveles de postura emprendedora como consecuencia de sus mayores recursos y capacidades existentes. Pues existe evidencia de empresas que a pesar de ser pymes logran convertirse en empresas altamente exportadoras o Born Global. De igual forma, dentro de este estudio se comprueba que altos niveles de I+D permiten establecer una mejor postura emprendedora e influenciar en los rendimientos de la empresa en los mercados internacionales.

En cuanto a las características externas, en este estudio, sobresale el rol de los niveles tecnológicos del sector como la principal característica de este constructo y su influencia en el logro de una fuerte postura emprendedora. Esto fue evidente en sectores intensivos en conocimiento e impone un referente de los niveles necesarios de tecnología para lograr competir dentro del sector en los mercados internacionales. Adicionalmente, este artículo aporta evidencia empírica en Colombia para la relación entre rivalidad sectorial y su asociación positiva con la actividad exportadora de una organización.

Por su parte, se comprueba la relación positiva de los recursos gubernamentales y la postura emprendedora, resaltando el papel de los recursos financieros como facilitador del desarrollo empresarial y que genera la necesidad que el empresario esté en contacto 
permanente con actores claves gubernamentales, a través de los acuerdos de financiación patrocinados por el gobierno y financiados con fondos públicos para la promoción de exportaciones y la sobrevivencia empresarial.

Este trabajo contribuye a analizar la postura emprendedora como factor moderador entre diversos factores internos y externos de las empresas que afectan el desempeño exportador. En este trabajo se ha intentado demostrar la importancia del emprendimiento, más específicamente, de una postura emprendedora asumida por las empresas como una serie de habilidades que desarrolla la empresa gracias a las características del empresario, a los factores internos de la empresa, a los factores externos y al apoyo del gobierno. Por tanto, los resultados obtenidos generan un panorama general de los principales aspectos que una pyme debe tener en cuenta si su objetivo es promover las exportaciones, focalizándose en el desarrollo de sus recursos internos y externos. Adicionalmente, siendo las pymes empresas que por su tamaño cuentan con ciertas limitaciones en materia de recursos para mantenerse en el mercado y acceder a mejores oportunidades, los resultados obtenidos muestran una guía a las empresas en aspectos como las características del personal contratado, la inversión de los recursos, y un mayor análisis de su entorno económico y social para poder identificar sus fortalezas y debilidades en el mercado. Adicionalmente, se muestra la importancia del apoyo gubernamental para incentivar la actividad exportadora dado que otorga un mayor soporte a las empresas para tomar sus decisiones de internacionalización, de allí la importancia de contar con políticas gubernamentales que apoyen el desarrollo tanto de las pymes como de las exportaciones.

Por último, es importante enfatizar que las conclusiones planteadas en este estudio se hacen sobre la base de una metodología de corte transversal y por tanto, no refleja los factores dinámicos presentes en toda organización. Sin embargo, sí permite hacer una contribución en el establecimiento de relaciones causales y mejorar la comprensión de las características de la postura emprendedora y su influencia en el desempeño exportador en Colombia. Para futuras investigaciones se podrían plantear nuevas hipótesis donde se analice si el desempeño exportador se encuentra determinado por otra cantidad de factores internos y externos a la organización que merecen ser incluidos en estudios posteriores, como por ejemplo, la cultura organizacional, el nivel de innovación del sector, la financiación por parte del sector privado, entre otros. Del mismo modo, merece una mayor atención un estudio independiente de la postura emprendedora donde se analice con mayor detenimiento sus antecedentes y consecuencias diferentes al desempeño exportador. 


\section{Referencias}

Aaby, N., and Slater, S. (1989). Management influences on export performance: A review of the empirical literature 1978-88. International Marketing Review, 6 (4), 7-26.

Acedo, F., and Jones, M. (2007). Speed of internationalization and entrepreneurial cognition: Insights and a comparison between international new ventures, exporters and domestic firms. Journal of World Business, 42, 236-252.

Albaum, G., Duerr, E., and Strandskov, J. (2001). International marketing and export management. London: FT Prentice Hall.

Alonso, J., y Donoso, V. (1998). Competir en el exterior. La empresa española y los mercados internacionales. Madrid: Icex.

Álvarez, L., Santos, M., y Vázquez, R. (2005). Escalas de medida del concepto de orientación al mercado: revisión crítica de su contenido y de sus prioridades psicométricas. ESIC Market, abril, 161-202.

Andersson, S. (2000). The internationalization of the firm from an entrepreneurial perspective. International Studies of Management \& Organization, 30 (1), 63-93.

Andersson, S., Gabrielsson, J., and Wictor, I. (2004). International activities in small firms: Examining factors influencing the internationalization and export growth of small firms. Canadian Journal of Administrative Sciences, 21 (1), 22-34.

Audretsch, D., Aldridge, T., and Oettl, A. (2006). The knowledge filter and economic growth: The role of scientist entrepreneurship. Discussion Papers on Entrepreneurship, Growth and Public Policy, 1106, 1-67.

Autio, E., Sapienza, H., and Almeida, J. (2000). Effects of age at entry, knowledge intensity, and imitability on international growth. Academy of Management Journal, 43 (59), 909- 924.

Autio, E., and Sapienza, H. (2000). Comparing process and born global perspectives in them international growth of technology-based new firms. Frontiers of Entrepreneurship Research. Babson College, 413-424.

Axinn, C. (1988). Export performance: Do managerial perceptions make a difference? International Marketing Review, 5 (2), 61-71.

Balabanis, G., and Katsikea, E.S. (2003). Being an entrepreneurial exporter: Does it pay? International Business Review, 12, 233-252.

Behyan, M. (2015). Analyzing factors affecting export performance of manufacturing firms. International Journal of Information Science and Management, 13 (Special Issue), 77-91.

Bell, J. (1995). The internationalization of small computer software firms: A further challenge to stage theories. European Journal of Marketing, 29 (8), 60-75.

Bell, J., McNaughton, R., Young, S., and Crick, D. (2003). Towards an integrative model of small firm internationalization. Journal of International Entrepreneurship, 1 (4), 339-362. 
Belso, J., (2003). Un análisis del proceso de internacionalización de las pequeñas y medianas empresas en la comunidad Valenciana: Modelo gradual versus acelerado. Revista Valenciana de Economía y Hacienda, 2 (8), 192-209.

Bleaney, M., and Wakelin, K. (2002). Efficiency, innovation and exports. Oxford Bulletin of Economics and Statistics, 64 (3), 3-15.

Blesa, A., y Ripollés, M. (2004). Orientación emprendedora, orientación al mercado y rendimiento empresarial. Revista europea de dirección y economía de la empresa , 5, 935-946.

Blomqvist, K., Hurmelinna-Laukkanen, P., Nummela, N., and Saarenketo, S. (2008). The role of trust and contracts in the internationalization of technology-intensive Born Globals. Journal of Engineering and Technology Management, 25 (1/2), 123-135.

Cadogan, J., Cui, C., Morgan, R., and Story, V. (2006). Factors facilitating and impeding the development of export market-oriented behavior: A study of Hong Kong manufacturing exporters. Industrial Marketing Management, 35, 634-647.

Cadogan, J., Cui, C., and Yeung Li, E. (2003). Export market-oriented behavior and export performance: The moderating roles of competitive intensity and technological turbulence. International Marketing Review, 20 (5), 493-513.

Cadogan, J., Diamantopoulos, A., and Siguaw, J. (2002). Export market-oriented activities: Their antecedents and performance consequences. Journal of International Business Studies, 33 (3), 615-626.

Cadogan, J., Paul, N., Salminen, R., Puumalainen, K., and Sundqvist, S. (2001). Key antecedents to export market-oriented behaviors: A cross-national empirical examination. International Journal of Research in Marketing, 18 (3), 261-282.

Cahill, D. (1996). Entrepreneurial orientation or pioneer advantage. Academy of Management Review, 21 (3), 603-606.

Cancino, C., y Bonilla, C. (2009). Los factores claves de una rápida internacionalización de las pequeñas y medianas empresas en Chile. Recuperado el 12 de agosto de 2009, de http:// mba.americaeconomia.com/biblioteca/papers/los-factores-claves-de-una-rapida internacionalizacion-de-las-pequenas-y-medianas.

Canon, T., and Wilis, M. (1983). The smaller firm in overseas trade. European Small Business Journal, 1 (3), 45-55.

Caruana, A., Morris, M., and Vella A. (1998). The effect of centralization and formalization on entrepreneurship in export firms. Journal of Small Business Management, 25 (2) 16-29.

Cavusgil, S., and Naor, J. (1987). Firm and management characteristics as discriminators of export marketing activity. Journal of Business Research, 15, 221-235.

Cavusgil, S., y Zou, S. (1994). Marketing strategy-performance relationship: An investigation of the empirical link in export market ventures. Journal of Marketing, 58, 1-21. 
Cavusgil, S. T. (1984). Differences among exporting firms based on their degree of Internationalization. Journal of Business Research, 12 (3), 195-208.

Cerrato, D. (2009). Does innovation lead to global orientation? Empirical evidence from a sample of Italian firms. European Management Journal, 27, 305-315.

Cooper, R. G. (1979). The dimensions of industrial new product success and failure. Journal of Marketing, 43 (2), 93-103.

Coviello, N., and McAuley, A. (1999). Internationalization and the smaller firm: A review of contemporary empirical research. Management International Review, 39 (3), 223-256.

Coviello, N., and Munro, H. (1997). Network relationships and the internationalization process of small software firms, International Business Review, 6 (4), 361-386.

Covin, J., and Slevin, D. (1988). The influence of organization structure on the utility of an entrepreneurial top management style. The Journal of Management Studies, 25 (3), 217-235.

Covin, J., and Slevin, D. (1989). Strategic management of small firms in hostile and benign environments. Strategic Management Journal, 10, 75-87.

Covin, J., and Slevin, D. (1991). A conceptual model of entrepreneurship as firm behavior. Entrepreneurship Theory and Practice, 16 (3), 7-24.

Cragg, P., and King, M. (1988). Organizational characteristics and small firms' performance revisited. Entrepreneurship Theory and Practice, 13 (2), 49-64.

Cyert, R., and March, J. (1963). A behavioral theory of the firm. New York: Prentice-Hall.

Czinkota, M., and Johnson, W. (1983). Exporting: Does sales volume make a difference? Journal of International Business Studies, spring/summer, 147-153.

Daniels, R. (2004). Negocios Internacionales. Bogotá: Prentice Hall.

De Clercq, D., Honig, B., and Martin, B. (2013). The roles of learning orientation and passion for work in the formation of entrepreneurial intention. International Small Business Journal, 31 (6), 652-676.

Del Barrio, S. y Luque, T. (2000). Análisis de ecuaciones estructurales. En Luque, T. Técnicas de análisis de datos en investigación de mercados, Madrid: Ediciones Pirámide.

Dian (2012). Boletín de Comercio Exterior, enero a diciembre de 2011-2012. Consulta en línea en www.dian.gov.co

Dimitratos P., and Plakoyiannaki, E. (2003). Theoretical Foundations of an International Entrepreneurial Culture. Journal of International Entrepreneurship, 1 (2), 187-215.

Ding, W., and Stuart, T. (2006). When do scientists become entrepreneurs? The social structural antecedents of commercial activity in the academic life sciences. American Journal of Sociology, 112 (1), 97-144.

Duncan, R. (1972). Characteristics of organizational environments and perceived environmental uncertainty. Administrative Science Quarterly, 3, 313-327. 
Emery, F., and Trist, E. (1965). The causal texture of organizational environments. Human Relations, 18, 21-31.

Escandón, D., y Hurtado, A. (2012). Características de los directivos de las empresas Born Global en Colombia. Entramado, 8 (2), 74-93.

Escandón, D., y Hurtado, A. (2013). Dinámica exportadora de las empresas Born Global en Colombia. Revista Finanzas y Política Económica, 5 (1), 107-138.

Escandón, D., y Hurtado, A. (2014). Factores que influyen en el desarrollo exportador de las pymes en Colombia. Estudios Gerenciales, 30 (131), 172-183.

Etchebarne, M., Geldres, V., y García Cruz, R. (2010). El impacto de la orientación emprendedora en el desempeño exportador de la firma, EsicMarket, 137, 193-220.

Evangelista, F. (1994). Export performance and its determinants: Some empirical evidence from Australian manufacturing firms. Advice International Marketing, 6, 207-229.

Fernández-Mesa, A., and Alegre, J. (2015). Entrepreneurial orientation and export intensity: Examining the interplay of organizational learning and innovation. International Businesshemm Review, 24 (1), 148-156.

Ferrier, J. (2001). Navigating the competitive landscape: The drivers and consequences of competitive aggressiveness. Academy of Management Journal, 44, 858-877.

Fillis, I. (2000). Being creative at the marketing/entrepreneurship interface: Lessons from the art industry. Journal of Research in Marketing and Entrepreneurship, 2 (2), 125-137.

Fornell, C., and Larcker, D. (1981). Evaluating structural equation models with unobservable variables and measurement error. Journal of Marketing Research, 27, 39-50.

Francioni, B., Musso, F., and Cioppi, M. (2015). Decision-maker characteristics and international decisions for SMEs. Management Decision, 53 (10), 2226-2249.

Frishammar, J., and Hörte, S. (2007). The role of market orientation and entrepreneurial orientation for new product development performance in manufacturing firms. Technology Analysis \& Strategic Management, 19 (6), 765-788.

Galbreath, J. (2005). Which resources matter the most to firm success? An exploratory study of resource-based theory. Technovation, 25, 979-987.

Gedik, S., Miman, M., and Serdar, M. (2015). characteristics and attitudes of entrepreneurs towards entrepreneurship. Procedia - Social and Behavioral Sciences, 195, 1087-1096.

Geldres, V., Etchebarne, M., y Bustos, L. (2009). Metodología DID Matching aplicada a la política pública de promoción de exportaciones. Revista de Administración, 16 (1), 59-90.

Gem Colombia (2011). Reporte GEM Colombia 2011. Recuperado de http://www.gemcolombia. org/images/Reporte\%202011.pdf.

Gençtürk, E., and Kotabe, M. (2001). The effect of export assistance program usage on export performance: A contingency explanation. Journal of International Marketing, 9 (2), 51-72. 
Gnyawali, D., and Fogel, D. (1994). Environments for Entrepreneurship development: Key Dimensions and Research Implications. Entrepreneurship, Theory and Practice, 18 (4), 43-62.

Gomez-Mejia, L. (1988). The role of human resources strategy in export performance: A longitudinal study. Strategic Management Journal, 9, 493-505.

Grinstein, A. (2008). The relationship between market orientation and alternative strategic orientations: A meta-analysis. European Journal of Marketing, 42 (1/2), 115-134.

Guth, W., and Ginsberg, A. (1990). Guest editors' introduction: Corporate entrepreneurship. Strategic Management Journal, 11, 5-15.

Hedlund, G., and Kverneland, A. (1985). Are strategies for foreign markets changing? The case of Swedish investment in Japan. International Studies of Management and Organization, 15 (2) 41-59.

Herron, L., and Robinson, R. Jr (1993). A structural model of the effects of entrepreneurial characteristics on venture performance. Journal of Business Venturing, 8 (3), 281-295.

Hollenstein, H. (2005). Determinants of International Activities: Are SMEs Different? Small Business Economics, 24 (5), 431-450.

Houthoofd, N., and Hendrickx, J. (2012). Export intensity and firm performance for family brewers. HUB Research Paper Economics \& Management, 25, 1-39.

Ibrahim, A., and Goodwin, J. (1986). Perceived causes of success in small business. Entrepreneurship Theory and Practice, 11 (2), 41-51.

Ismail, Md.D., Isa, A.Md., and Ali, M.H. (2013). Insight into the relationship between entrepreneurship orientations and performance: The case of SME exporters in Malaysia. Journal Pengurusan, 38, 63-73.

Jain, S. (1989). Standardization of international marketing strategy: Some research hypotheses. Journal of Marketing, 53 (1), 70-79.

Jap, S. (1999). Pie-expansion efforts: Collaboration processes in buyer-seller relationships. Journal of Marketing Research, 36 (4), 461-475.

Jaworski, B., and Kohli, A. (1993). Market orientation: Antecedents and consequences. The Journal of Marketing, 57 (3), 53-70.

Jennings, D., and Lumpkin, J. (1989). Functionally modelling corporate entrepreneurship: An empirical integrative approach. Journal of Management, 15 (3), 485-502.

Johanson, J., and Vahlne, J. (1977). The internationalization process of the firm: A model of knowledge development and increasing foreign market commitment. Journal of International Business Studies, 8 (1), 23-32.

Johanson, J., and Vahlne, J. (1990). The mechanism of internationalization. International Marketing Review, 7 (4), 11-24. 
Johanson, J., and Vahlne, J. (2003). Building a model of firm internationalisation. In A. Blomstermo, and D. Sharma (eds.). Learning in the Internationalisation Process of Firms, (pp. 3-15). Cheltenham: Edward Elgar

Johanson, J., and Wiedersheim-Paul, F. (1975). The internationalization of the firm: Four Swedish cases. Journal of Management Studies, 12 (2), 305-322.

Jolly, V Alahulta, M., and Jeanet, J. (1992). Challenging the incumbents: How high technology start-ups complete globally. Journal of Strategic Change, 1, 71-82.

Johnson, J., and Arunthanes, W. (1995). Ideal and actual product adaptation in US exporting firms: Market - related determinants and impact on performance, International Marketing Review, 12 (3), 31-46.

Karra, N., Phillips, N., and Tracey, P. (2008). Building the Born Global firm. Developing entrepreneurial capabilities for international new venture success. Long Range Planning, 41, 440-458.

Katsikeas, C., Piercy, N., and Ioannidis, C. (1996) Determinants of export performance in a European context, European Journal of Marketing, 30 (6), 6-35.

Keats, B., and Bracker, J. (1988). Toward a theory of small firm performance: A conceptual model. Entrepreneurship Theory and Practice, 12 (4), 41-59.

Kim, J.-J., and Hemmert, M. (2016). What drives the export performance of small and medium-sized subcontracting firms? A study of Korean manufacturers. International Business Review, 25 (2), 511-521.

Kim, Y.G., and Suh, J. (2015). What determines small champions' export performance? Evidence from Korea firm-level data. Asian Economic Papers, 14 (2), 138-155.

Knight, G. (2000). Entrepreneurship and marketing strategy: the SME under globalization. Journal of International Marketing, 8 (2), 12-32.

Knight, G., and Cavusgil, S. (2004). Innovation, organizational capabilities, and the born-global firm. Journal of International Business Studies, 35 (2), 124-141.

Knight, G., and Cavusgil, S. (1996). The Born Global firm: A challenge to traditional internationalization theory. Advances in International Marketing, 8, 11-26.

Knudsen, T., and Madsen, T. (2002). Export strategy: A dynamic capabilities perspective. Scandinavian Journal of Management, 18, 475-502.

Kotabe, M., and Czinkota, M. (1992). State government promotion of manufacturing exports: A gap analysis. Journal of International Business Studies, 23 (4), 637-658.

Kuivalainen, 0., Sundqvist, S., and Servais, P. (2007). Firms' degree of born-globalness, international entrepreneurial orientation and export performance. Journal of World Business, 42, 253-267. 
Laanti, R., Gabrielsson, M., and Gabrielsson, P. (2007). The globalization strategies of business-to-business born global firms in the wireless technology industry. Industrial Marketing Management, 36, 1104-1117.

Lacouture, M. (2015). ¿Qué tanto han aprovechado las pymes el comercio? Revista Dinero. Recuperado el 15 de septiembre de 2015 de http://www.dinero.com/edicion-impresa/caratula/articulo/que-tanto-han-aprovechado-pymes-comercio-exterior-colombia/213538.

Lages, L., and Montgomery, D. (2005). The relationship between export assistance and performance improvement in Portuguese export ventures: An empirical test of the mediating role of pricing strategy adaptation. European Journal of Marketing, 39 (7/8), 755-784.

Laguir, I., and Den Besten, M. (2016). The influence of entrepreneur's personal characteristics on MSEs growth through innovation. Applied Economics, 1-18. Article in Press.

Lee, W., and Brasch, J. (1978). The adoption of export as an innovative strategy. Journal of International Business Studies, 9 (1), 85-93.

Leonidou, L., Katsikeas, C., and Piercy, N. (1998). Identifying managerial influences on exporting: Past research and future directions. Journal of International Marketing, 6 (2), 74-102.

Li, Y., Zhao, Y., Tan, J., and Liu, Y. (2008). Moderating effects of entrepreneurial orientationperformance linkage: Evidence from Chinese small firms. Journal of Small Business Management, 46 (1), 113-133.

Lu, J., and Beamish, P. (2001). The internationalization and performance of SMEs. Strategic Management Journal, 22 (6/7), 565-586.

Lumpkin, G., and Dess, G. (1996). Clarifying the entrepreneurial orientation construct and linking it to performance: Patterns in one industry. Academy of Management Review, 21 (1), 135-172.

Lyon, D., Lumpkin, G., and Dess, G. (2000). Enhancing entrepreneurial orientation research: Operationalizing and measuring a key strategic decision making process. Journal of Management, 26, 1055-1085.

Madsen, T. (1998). Executive insights: Managerial judgment of export performance, Journal of International Marketing, 6 (3), 82-93.

Madsen, T., and Servais, P. (1997). The internationalization of Born Globals: An evolutionary process? International Business Review, 6, 561-583.

Matt, M., and Wolff, S. (2004). Incentives, coordination and learning in government- sponsored vs. spontaneous inter-firm research cooperation. International Journal of Technology Management, 27 (8), 694-711.

Mazzarol, T., Volery, T., Doss, N., and Thein, V. (1999). Factors influencing small business startups. International Journal of Entrepreneurial Behavior and Research, 5 (2), 48-63.

McDougall, P., and Oviatt, B. (2000). International entrepreneurship: The intersection of two research paths. Academy of Management Journal, 43, 902-908. 
Miller, D. (1983). The correlates of entrepreneurship in three types of firms. Management Science, 29 (7), 770-791.

Miller, D., Droge, C., and Tolouse, J. (1988). Strategic process and content as mediators between organizational context and structure. Academy of Management Journal, 31, 554-569.

Miller, D., and Friesen, P. (1982). Innovation in conservative and entrepreneurial firms: Two models of strategic momentum. Strategic Management Journal, 3 (1), 1-25.

MinCIT (2014). Evolución de las empresas y productos importados, 1991-2013. Documentos 0EE18, marzo.

Moen, 0., and Servais M. (2002). Born Global or Gradual Global? Examining the export behavior of small and medium-sized enterprises. Journal of International Marketing, 10 (3), 49-72.

Moini, A. (1998). Small firms exporting: How effective are government export assistance programs? Journal of Small Business Management, 36 (1), 1-15.

Moori, V., Rodriguez, A., Yoguel, G., y Granados, A. (2005). Perfil de la pyme exportadora exitosa: El caso colombiano. FUNDES Internacional.

Moori, V., Yoguel, G., Milesi, D, y Gutierrez, I. (2004). Buenas prácticas de las pyme exportadoras exitosas PYMEX: el caso chileno. FUNDES Internacional.

Morris, M., and Paul. G. (1987). The relationship between entrepreneurship and marketing in established firms, Journal of Business Venturing, 2, 247-259.

Morrison, A., and Roth, K. (1992). A taxonomy of business level strategies in global industries. Strategic Management Journal, 13, 399-418.

Mostafa, R.H., Wheeler, C., and Jones, M.V. (2005). Entrepreneurial orientation, commitment to the Internet and export performance in small and medium sized exporting firms. Journal of International Entrepreneurship, 3 (4), 291-302.

Oviatt, B., and McDougall, P. (1995). Global start-ups: Entrepreneurs on a wold-wide stage. Academy of Management Executive, 9 (2), 30-43.

Oviatt, B., and Mcdougall, P. (2005). Defining international entrepreneurship and modeling the speed of internationalization. Entrepreneurship Theory and Practice, 29 (5), 537-553.

Ozsomer, A., and Gençtürk, E. (2003). A resource based model of market learning in the subsidiary: The capabilities of exploration and exploitation. Journal of International Marketing, $11(3), 1-29$.

Pauwels, P., and Matthyssens, P. (1999). A strategy process perspective on export withdrawal. Journal of International Marketing, 7 (3), 10-37.

Peng, M. (2001). The Resource-based view and international business. Journal of Management, 27 (6), 803-829.

Prahalad, C., and Hamel, G. (1990). The core competence of the corporation. Harvard Business Review, 68 (3), 79-91. 
Prietula, M. J., and Watson, H. S. (2000). Extending the Cyert March duopoly model: Organizational and economic insights. Organization Science, 11 (5), 565-585.

Reid, S. (1983). Managerial and firm influences on export behavior. Journal of the Academy of Marketing Science, 11 (3), 323-332.

Reynolds, P., Hay, M., Bygrave, W., Camp, S., and Autio, E. (2000). Global Entrepreneurship Monitor 2000 Executive Report: Babson College, Kauffman Center for Entrepreneurial Leadership, and London Business School.

Rialp, A. y Rialp, J. (2005). Las formas actuales de penetración y desarrollo de los mercados internacionales: caracterización, marcos conceptuales y evidencia empírica en el caso español. Claves de la Economía Mundial. Madrid: ICEX.

Rialp, A., Rialp, J., and Knight, G. (2005). The phenomenon of early internationalizing firms: what do we know after a decade (1993-2003) of scientific inquiry? International Business Review, 14 (2), 147-166.

Rialp, A., and Rialp, J. (2007). Faster and more successful exporters: An exploratory study of born global firms from the resource-based view. Journal of Euromarketing, 16 (1-2), 71-86.

Rialp-Criado, A., Rialp-Criado, J., and Knight G. (2002). The phenomenon of international new ventures, global start-ups, and born-globals: What do we know after a decade (1993-2002) of exhaustive scientific inquiry? Universidad Autónoma de Barcelona.

Rosson, P., and Seringhaus, F. (1991). Export development and promotion. The role of public organizations. London: Kluwer Academic Publishers.

Schlegelmilch, B., and Crook, J. (1988). Firm-level determinants of export intensity. Managerial and Decision Economics, 9, 291-300.

Shane, S., and Khurana, R. (2003). Bringing individuals back in the effects of career experience on new firm founding. Industrial and Corporate Change, 12 (3), 519-543.

Shane, S., and Venkataraman, S. (2000). The promise of entrepreneurship as a field of research. Academy of Management Review, 25, 217-226.

Shrader, R., Oviatt, B., and Mcdougall, P. (2000). How new ventures exploit trade-offs among international risk factors: Lessons for the accelerated internationalization of the 21st century. Academy of Management Journal, 43 (6), 1227-1247.

Sinha, T. N. (1996). Human factors in entrepreneurship effectiveness. The Journal of Entrepreneurship, 5 (1), 23-39.

Sousa, C., Martínez-López, F., and Coelho, F. (2008). The determinants of export performance: A review of the research in the literature between 1998 and 2005. International Journal of Management Reviews, 10 (4), 343-374.

Starbuck, W. (1975). Organizations and their environments. In M. Dunnette (ed.), Handbook of Organizational Psychology (pp. 1069-1123). Chicago: Rand McNally.

Storey, D. (1994). Understanding the Small Business Sector. London: Routledge. 
Thompson, J. (1967). Organizations in action. New York: McGraw-Hill.

Westhead, P., Binks, M., Ucbasaran, D., and Wright, M. (2002). Internationalization of SMEs: A Research Note. Journal of Small Business and Enterprise Development, 9 (1), 38-48.

Westhead, P., Wright, M., and Ucbasaran, D. (2001). The Internationalization of New and Small Firms: A Resource-based View. Journal of Business Venturing, 16 (4), 333-358.

Yañez, C.J., Magnier, A., Ramirez, M.A. (2008). Local governance as government- business cooperation in western democracies: analyzing local and inter-governmental effects by multi-level comparison. International Journal of Urban and Regional Research, 32 (3), 531-547.

Zahra, S. (1991). Predictors and financial outcomes of corporate entrepreneurship: An exploratory study. Journal of Business Venturing, 6 (4), 259-286.

Zahra, S. (1993). A conceptual model of entrepreneurship as firm behavior: A critique and extension. Entrepreneurship Theory and Practice, 17 (4), 5-22.

Zahra, S., Neubaum, D., and Huse, H. (1997). The effect of the environment on export performance among telecommunications new ventures. EntrepreneurTheory \& Practice, 22 (1), 25-46.

Zahra, S., and Garvis, D. (2000). Entrepreneurship and firm performance: The moderating effect of international environmental hostility. Journal of Business Venturing, 15 (5), 469-492.

Zhang, M., Sarker, S., and Sarker, S. (2013). Drivers and export performance impacts of IT capability in 'born-global' firms: A cross-national study. Information Systems Journal, 23 (5), 419-443.

Zou, S., and Stan, S. (1998). The determinants of export performance: A review of the empirical literature between 1987 and 1997. International Marketing Review, 15 (5), 333-356. 
